in about the third or fourth week. She was then observed to have internal strabismus of the left eye, caused by paralysis of the left external rectus muscle. There were no cerebral symptoms, no headache nor any other paralysis, no rigidity nor signs of meningeal involvement.

Dr. Veasey examined the patient later and reported paralysisi of the left sixth nerve. The patient saw double images on following the moving object to the left beyond, or a little beyond, the middle line, and the images tended to get further apart. There was no paralysis of accommodation, nor of any outer muscles of the eye. The fundus was normal. This patient had been under treatment for her eyes at the dispensary of the hospital before her typhoid fever, but at that time had no paralysis of her sixth nerve; so the onset of the trouble during the typhoid fever is quite clear. Since leaving the hospital, about ten days ago, the paralysis has decreased, and while still apparent it is not so noticeable as it was formerly. This, of course, is of good augury, and shows that the prognosis is favorable, just as in cases of diphtheritic paralysis, and that the patient will probably make a satisfactory recovery. Such cases must be very rare. This is the only one of the kind Dr. Lloyd had ever seen, and the literature is scanty. He had found only one reference to a somewhat similar case, but associated with double ptosis. Dr. de Schweinitz has stated that paralysis of accommodation after typhoid fever is not uncommon, but that other ocular palsies are very rare. The analogy of this case to some cases of postdiphtheritic paralysis will probably strike everybody, but in post-diphtheritic paralysis there is usually paralysis of accommodation, as well as nasal speech, dysphagia and loss of power in some of the extremities with abolition of the knee-jerks, none of which symptoms have been present in this patient. Besides, in this patient, who was under careful observation during most of her fever, there was no evidence of a diphtheritic process. Neither had there been any polyuria, as is seen in some cases of sixth nerve paralysis. Nor was it possible to make out a specific history.

\title{
NEW CLINICAL SYMPTOMS IN HEMIPLEGIA AND TABES DORSALIS.
}

By Drs. T. H. Weisenburg and C. C. Manger.

In a series of hemiplegias it was noted that the palpebral fissure on the hemiplegic side was larger than on the sound side. This symptom was looked for in about ninety cases and found in about eighty. It is probable that this was due to the drooping of the lower lid. This sign has not heretofore been noted, and is of some importance in the instant recognition of paralysis. It is, of course, also present in peripheral facial palsy.

For some time Dr. Weisenburg has noticed that patients with tabes dorsalis have a remarkable similarity of facial expression. So much so that it has been possible in many instances to recognize patients with this disease on sight. The composite picture shows this very easily. There is a paleness of the face and puckering of the brow and drooping of the upper lid and the corners of the mouth, and a peculiar expression around the eyes which is rather difficult to describe but easy to recognize. Besides, in a series of thirty-five cases, all men, the eyes had been found to be either blue or gray. This does 
not hold true in women. The occurrence of blue or gray eyes in such a large percentage of cases examined is rather important and while this number is very small, still it is suggestive.

Dr. Charles W. Burr said any study of a possible relation between the color of the eyes and the occurrence of tabes to be of any value would have to include a very large number of patients. It was necessary also that the race of the patients and not merely their place of birth should be considered. Dark-eyed people were not as common at Blockley as light-eyed.

Dr. W. G. Spiller said that the widening of the palpebral fissure in hemiplegia, to which Dr. Weisenburg alluded, brought up the interesting question of paresis in the upper branch of the facial nerve distribution in hemiplegia. When Dr. Spiller was in Vienna in 1893 the paresis of the upper part of the face in hemiplegia was well recognized. In almost every case of hemiplegia where the face is implicated there is at first some involvement in the distribution of the upper branch of the seventh nerve. It is usually of transitory duration. The widening of the palpebral fissure to which Dr. Weisenburg alluded, probably depends on paresis of the orbicularis palpebrarum muscle.

Dr. Sailer called attention to the value of composite photographs for the purpose of determining the physiognomy of disease. The opportunities at Blockley for making such photographs are excellent, and they might be of great service in the conditions under discussion, that is, tabes dorsalis and hemiplegia. In the latter the difference in the palpebral fissure would probably be more accentuated in such a photograph than in any individual case.

Dr. D. J. McCarthy thought the involvement of the lids was due to secondary contracture pulling down the inferior lid. Dr. MeCarthy also said that cases of tabes in the negro had been frequently reported to the Society. In all these cases, of course, the eyes were dark.

Dr. Spiller said in regard to the patients presented, they were old, and it is common to find in the aged a little drooping of the lower lid in paresis of the upper branch of the facial because of the loss of the elasticity of the skin.

Dr. Weisenburg in closing agreed with Dr. Butr that 35 cases of tabes in which blue eyes constantly occurred did not demonstrate much, but he thought that the fact that it occurred in so many cases was rather interesting. In the study of these cases, the race of the patients was considered, but there was no definite relation found. Dr. Weisenburg's main object in bringing this matter before the Society was, if possible, to obtain further information upon the subject, and also to stimulate further observations in the color of the eyes in tabetic cases.

Dr. Weisenburg did not agree with Dr. Spiller that the widening of the palpebral fissure was the result of the paresis in the upper part of the face, but thought that it was due to the paresis of the lower eyelid. He did not agree with Dr. McCarthy that this sign is due to secondary contracture as in secondary contracture we should have the opposite condition, that is lessening in the width of the palpebral fissure.

\section{INCIPIENT TABES WITH SEVERE PAINS IN THE NECK. By Dr. W. G. Spiller.}

The patient was a male, 55 years of age. He had ataxia of gait, Romberg sign, difficulty at times in urination, numbness of the hands, very 